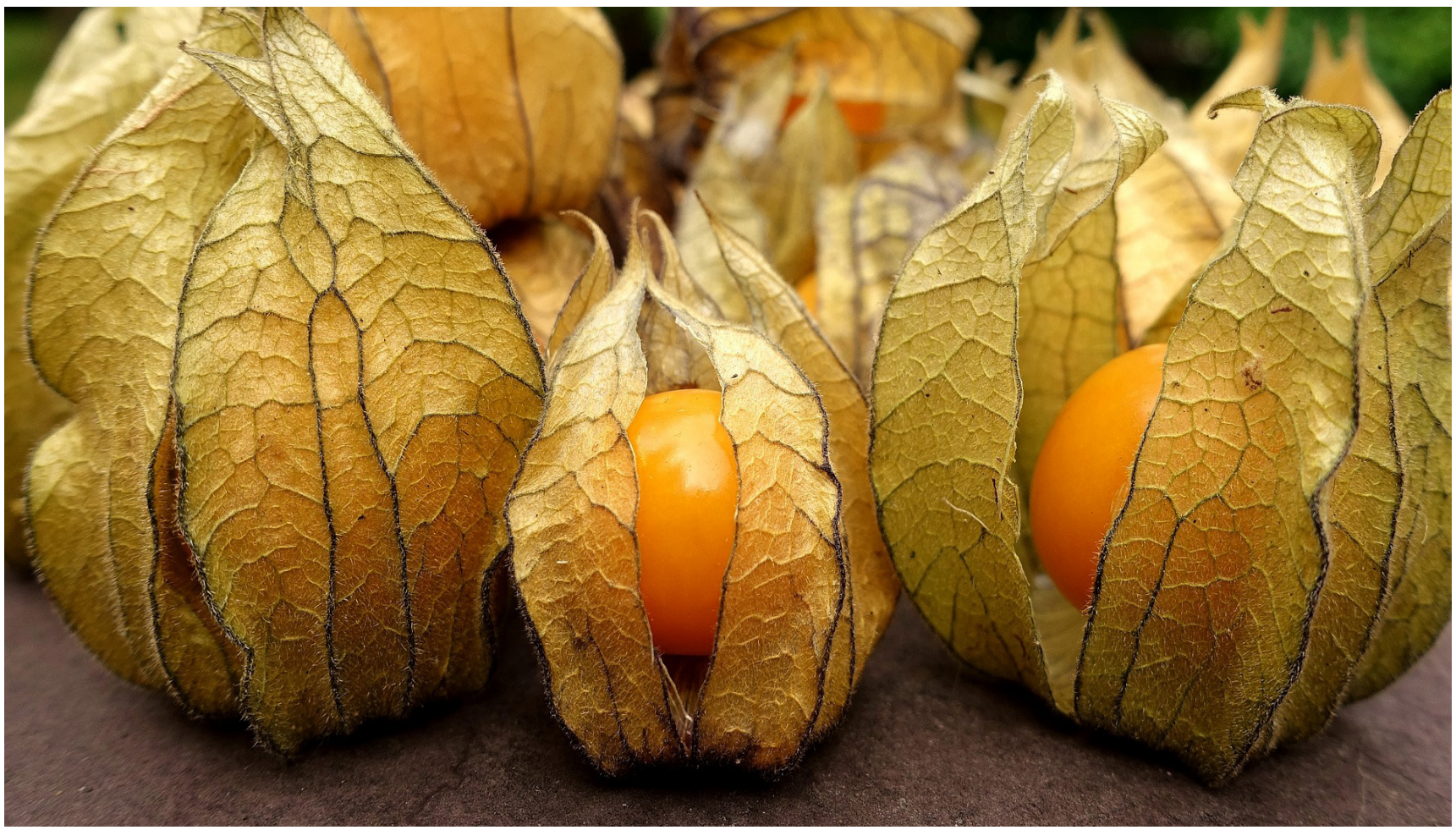

\title{
Efecto del pretratamiento con ultrasonido en las cinéticas de secado convectivo de la uchuva (physalis peruviana)
}

\section{Effect of ultrasound pretrating in convective drying kinetics of la uchuva (physalis peruviana)}

\section{Yesenia Campo-Vera}

Magister en Cienciay Tecnología de Alimentos, Directora de investigación, Grupo de investigacion en Ciencia, Tecnologia e Innovacion, yesenia. campo.vera@gmail.com, orcid: https://orcid.org/0000-0003-4658-8113, Instituto Superior de Educación Rural, Pamplona, Colombia

\section{Alfredo Ayala-Aponte}

Doctor en Ciencia y Tecnología de los Alimentos, Grupo de Investigación en Ingeniería de los Procesos Agroalimentarios y Biotecnológicos, alfredo.ayala@correounivalle.edu.co, Orcid: https://orcid.org/00000003-0310-3577, Universidad del Valle, Cali, Colombia

\section{Mauricio Contreras-Lozano}

Maestría en Ingenieria Industrial, Grupo de investigacion en Ciencia, Tecnologia e Innovacion, mauriciocontreras.docente@iser.edu.co, Orcid: https://orcid.org/0000-0003-1931-6278, Instituto Superior de Educación Rural, Pamplona, Colombia 
Resumen

El ultrasonido (US) es la práctica de innovación más utilizada en la manufactura alimentaria. Objetivo: En este trabajo se valuó el resultado del procedimiento con US $\left(40 \mathrm{KHz} / 130 \mathrm{~W} / 30^{\circ} \mathrm{C}\right.$ 110, 20 y $30 \mathrm{~min}$ ) en el secado por conducción a $60^{\circ} \mathrm{C} / 2 \mathrm{~m} / \mathrm{s}$ de la uchuva (Physalis peruviana). Metodología: Se manejó un patrón difusional para relatar las cinéticas de secado y cuantificar la influencia del US en la difusividad efectiva de agua. Resultados: Tomando en consideración que el US ascenso significativo $(p>0.05)$ la rapidez de secado en todos los ejemplares tratados con una reducción promedio del $38 \%$ en el tiempo de secado con respecto al tratamiento control; alcanzando perder peso del $78 \%$ en relación al peso inicial (4.21 a 0.93g.). Conclusiones: El modelo Logarítmico es el más conveniente para pronosticar las curvas experimentales de secado de la uchuva y reveló que el estudio de US creció tanto la difusividad positiva y el coeficiente de transmisión de masa, como confirman los valores del porcentaje de varianza explicada de 97.3 a $99.5 \%$.

Palabras clave: Difusividad de agua, Physalis peruviana, secado, uchuva, ultrasonido.
Abstract

Ultrasound (US) is the most widely used innovation practice in food manufacturing. Objective: In this work the result of the procedure with US $(40 \mathrm{KHz} / 130 \mathrm{~W}$ / 30 - C / 10, 20 and $30 \mathrm{~min}$ ) in the drying by conduction at $60^{\circ} \mathrm{C} / 2 \mathrm{~m} / \mathrm{s}$ of the cape gooseberry (Physalis peruviana) was evaluated - Methodology: A diffusion pattern was used to report the drying kinetics and quantify the influence of US on the effective diffusivity of water. Results: Taking into consideration that US significantly increased ( $p>0.05)$ the speed of drying in all treated specimens with an average reduction of $38 \%$ in drying time with respect to the control treatment; achieving to lose weight of $78 \%$ in relation to the initial weight (4.21 to 0.93g.). Conclusions: The Logarithmic model is the most appropriate to predict the experimental drying curves of cape gooseberry and revealed that the US study increased both the positive diffusivity and the mass transmission coefficient, as confirmed by the values of the percentage of explained variance of 97.3 to $99.5 \%$.

Keywords: Water diffusivity, Physalis peruviana, drying, gooseberry. ultrasound. 


\section{4}

\section{Introducción}

El secado es una técnica clásica que ayuda a la preservación de alimentos, es de vital beneficio para de disminuir el peso para su transporte y reduce el espacio para su almacenaje [1]. El secado aumenta la durabilidad de los alimentos al disminuir la actividad de agua, restringiendo la gestión microbiológica y los cambios fisicoquímicos que se originan en el almacenaje [2]. En el proceso de secado, pueden originar cambios físicos, estructurales, químicos, nutricionales en los vegetales y que logran afectar las propiedades de calidad como color, textura, valor nutricional y sabor [3].

La práctica que se ajusta al secado en bajas temperaturas es la liofilización, es donde se consiguen productos de buena calidad; pero con tiempos muy prolongados en el proceso, mayores a los del secado de aire caliente $[4,5]$. El secado es un paso complicado en el que causa una transmisión sincrónica de materia y de energía, la modelización forma un instrumento necesario para estudiar estos métodos y la atribución de los escenarios de manipulación [6], [7]; la transmisión de los compuestos dentro del alimento se promueve exclusivamente por las subidas de humedad, a lo que se llama mecanismo difusivo [8]. Así, la modelización de las cinéticas de secado consiente lograr medidas como la difusividad segura del producto, cuantificación básica para estimular la perfección el proceso de secado [9].

Inexplorados tratamientos anteriores al proceso de secado, se fundamentan en la manejo químico que ha alcanzado reducir los periodos de secado [6], [10]; actualmente, el ultrasonido se aplica (US) en el secado por aire caliente logrando la disminución del lapso de proceso y el gasto de energía en la operación, al lograr temperaturas menores convenientes para el secado [11], [12]. Asimismo, ha confirmado que crece la tasa de transmisión de masa durante el secado [6],[12],[13].

El acelerado incremento de la solicitud de la fabricación de la uchuva (Physalis peruviana) se solidariza con sus particularidades nutracéuticas y medicinales. Sin embargo, un aspecto que entorpece su mercadeo es por ser un producto perecedero. La uchuva, es un fruto originario de Colombia que se describe por el beneficioso contenido de vitamina $\mathrm{A} y$ $C$, hierro, zinc, fósforo y potasio y en virtud de su contenido de vitamina A se le conoce como un fruto carotenógeno [14], [15]. El sentido del proyecto fue calcular el resultado de la concentración de ultrasonidos $40 \mathrm{KHz} / 130 \mathrm{~W}$ a $30^{\circ} \mathrm{C}$ durante 10,20 y 30 min sobre las cinéticas del secado convectivo de la uchuva (Physalis peruviana).

\section{Materiales y métodos}

Preparación de la materia prima. Las uchuvas (cultivada en el municipio de Pamplona Norte de Santander) fueron obtenidas en un mercado minorista de la ciudad, con un peso promedio entre 4 a $6 \mathrm{~g}$, de formas semiesféricas. De acuerdo a la carta de color de la norma técnica colombiana, se utilizaron uchuvas con un estado de madurez entre los colores 3 y 5 [16]. Las frutas se lavaron con agua a presión y sanitizadas con una solución de hipoclorito de sodio (150 ppm) durante $3 \mathrm{~min}$. Aumentando por triplicado la humedad de las frutas enteras en estado fresco y deshidratado.

Tratamiento ultrasonido. Se utilizó un dispositivo BRANSON 3800, Modelo M $3800 \mathrm{H}$ $(40 \mathrm{KHz}$ ) y como medio de transferencia agua desionizada, las muestras (350 gr de uchuva) se volvieron someter a repetición de ultrasonido de $40 \mathrm{KHz} / 130 \mathrm{~W}$ a $30^{\circ} \mathrm{C}$ por un periodo 10,20 y $30 \mathrm{~min}$; como muestra control se empelaron uchuvas sin US. 
Secado convectivo. Se acordó que la cinética de secado se llevara a cabo en un secador convectivo de bandejas PS-SE-001/PE, Generatoris-México, usando una rapidez de aire de $2 \mathrm{~m} / \mathrm{s}$ y temperatura de $60^{\circ} \mathrm{C}$. Se tomaron tiempos inicialmente cada diez min durante las primeras $3 \mathrm{~h}$, posteriormente se tomaron cada 30 min hasta peso constante [2], [17].

Cálculo de energía de activación y del coeficiente de difusividad efectiva. En el presente experimento, la relación de humedad (MR) se utilizó como variable independiente (Eq. 1), esto corresponde satisfactoriamente a la humedad de la muestra de tiempo actual con el contenido de humedad en equilibrio [18]. Para detallar matemáticamente las cinéticas de secado se utilizó la ecuación integrada de la segunda ley de Fick utilizado durante períodos prolongados de tiempo (Eq. 2) y geometría esférica en una dimensión (Eq. 3) [19], [20].

$$
\begin{gathered}
M R=\frac{X w t-X w e}{X w o}-X w e \\
M R=\frac{6}{\pi^{2}} \sum_{j=1}^{\infty} \frac{1}{j^{2}} \exp \left[\frac{-j^{2} D_{\text {eff } \pi^{2} t}}{r^{2}}\right] \\
M R=\frac{6}{\pi^{2}} \exp \left[\frac{-D \text { effe } \pi^{2} t}{r^{2}}\right]
\end{gathered}
$$

Dónde: $X_{w t}$ : contenido de humedad en tiempo real (kg w/kg m.s.); $X_{\text {wo }}$ : contenido de humedad inicial (kg w/kg m.s); $X_{w e}$ : contenido de humedad en equilibrio (kg w/kg m.s); J: número de términos; $\mathrm{T}$ : tiempo (min); $\mathrm{R}$ es el radio de la muestra $(\mathrm{m})$; Deff: difusividad efectiva del agua $\left(\mathrm{m}^{2} / \mathrm{s}\right)$.

Generalmente, el predominio de secado en la temperatura sobre el coeficiente de difusividad positiva del agua en los alimentos sigue un estilo tipo Arrhenius, la reciprocidad explicada por la ecuación (4) y al simbolizar el Deff vs $1 / \mathrm{T}$ se logra una trazo recto cuya pendiente se consigue la Ea y de la ordenada al inicio se obtiene el factor de Arrhenius (Do).

$$
D e f f=D O \exp \frac{E a}{R T}
$$

Dónde: D0 es el factor de Arrhenius, $R$ es la constante universal de los gases y $\mathrm{T}$ es la temperatura absoluta.

Modelo Matemático. Se manejaron cinco diferentes tipos matemáticos de capa fina de los más manejados en el lenguaje matemático para constituir la cinética de secado experimental. La Tabla 1 muestra las expresiones de los modelos seleccionados:

En estos modelos, la variable dependiente es MR representa la relación de humedad (MR = $\mathrm{M} / \mathrm{MO}$ ). Dónde: $\mathrm{M}$ es la humedad del producto en un tiempo determinado, M0 la humedad en tiempo inicial, $\mathrm{t}$ es el tiempo de secado y $\mathrm{k}, \mathrm{n}$, a, b, c, g y h son parámetros o constantes de los modelos.

Tabla 1. Modelos matemáticos utilizados por diferentes autores en el estudio el comportamiento de secado.

\begin{tabular}{cllc}
\hline Eq. & Nombre & Ecuación modelo & Referencias \\
\hline 5 & Newton & MR $=\exp (-\mathbf{k t})$ & {$[21]$} \\
\hline 6 & $\begin{array}{l}\text { Henderson } \\
\text { Pabi }\end{array}$ & and & MR $=\mathbf{a} * \exp (-\mathrm{kt})$ \\
\hline 7 & Page & MR $=\exp (-\mathbf{k t n})$ & {$[22]$} \\
\hline 8 & Page Modificada & MR $=\exp (-\mathbf{k t}) \mathbf{n}$ & {$[24]$} \\
\hline 9 & Logarítmico & MR $=\mathbf{a} * \exp (-\mathbf{k t})+\mathbf{c}$ & {$[25]$} \\
\hline
\end{tabular}

Ajuste del modelo. En la cinética de secado, la difusividad segura se identificó acordando los modelos difusivos a los datos de experimento. La caracterización se llevó a cabo mediante la técnica de optimización del Gradiente Reducido. Generalizado (GRG), se estableció el ajuste a partir del porcentaje de varianza explicada (\%VAR, Eq.4) [2].

$$
\% V A R=1-\frac{s_{x y}^{2}}{s_{y}^{2}} * 100 \quad(\text { Eq. } 10)
$$

Dónde: Sxy y Sy son la desviación estándar de la estimación y de la muestra, respectivamente. 


\section{6}

Análisis estadístico. Las derivaciones obtenidas fueron tratados estadísticamente significativa a un nivel de 95\% ( $P<0.05)$, a fin de entender de manera explicita los resultados del ANOVA se utilizó la prueba post hoc de Diferencias Mínimas Significativas (DMS), con un software de estadísticas SPSS versión 22.0.

Para estimar el ajuste de los modelos matemáticos con los datos experimentales, se recurrió a los siguientes coeficientes estadísticos: el coeficiente de determinación (R2), CHI-cuadrado (X2) y suma de los errores al cuadrado (SSE). Los ajustes más predominantes fueron aquellos modelos que presentaron los valores más bajos de X2, SSE y los valores más altos de R2 [26].

$$
\begin{gathered}
X^{2}=\frac{\sum_{i=1} N\left(M R_{\text {expi } i}-M R_{\text {pre } i}\right)^{2}}{N-z} \quad(E q .11) \\
S S E=\frac{1}{N} * \sum_{i=1} N\left(M R_{\text {exp }, i}-M R_{\text {pre }, i}\right)^{2}(E q .12)
\end{gathered}
$$

MRexp,i personifican la correspondencia de humedad lograda experimentalmente, MRpre,i son los pronósticos hechos por los modelos matemáticos, $\mathrm{N}$ es el número de datos y $\mathrm{z}$ el número de constantes. Los cálculos y ajustes de los análisis estadísticos se ejecutaron con el software Microsoft Excel 2010.

Resultados y discusión

Secado convectivo. La figura 1 muestra las curvasexperimentales desecadodelasuchuvas pretratadas con US y la muestra control. La humedad inicial de la uchuva fue de $5.86 \pm$ $0,17 \mathrm{~kg} \mathrm{~W} / \mathrm{kg}$ m.s., similares a los obtenido por Duque [27] de $5,15 \pm 0,10 \mathrm{~g}$ agua/g $\mathrm{m} \mathrm{s}$. En las etapas iniciales del secado se observa una rápida eliminación de humedad de la fruta y en las etapas posteriores disminuye con el aumento del tiempo del proceso, debido a que el agua residual está fuertemente adherida en la fase solida de la uchuva formando la monocapa [28].
Este comportamiento es típico en el secado de frutas y vegetales con y sin US, como los reportados en diferentes productos como en uchuva [20], [29],[30], pitahaya amarilla [31], berenjena [32]. Los tratamientos con estudio de US mostraron y revelaron $(p<0.05)$ mayores pérdidas de humedad en menor tiempo de proceso respecto al tratamiento control (sin US); siendo el tratamiento con 30 min de aplicación de US el que presentó las mayores pérdidas para un mismo tiempo de secado, seguido de los tratamientos con aplicación 10 y 20 min de US que presentaron comportamiento similar $(p>0.05)$.

Las muestras tratadas con 30 min de US alcanzaron una humedad de $1,15 \mathrm{~kg} / \mathrm{kgm} . \mathrm{s}$ aproximadamente a los $210 \mathrm{~min}$, mientras que las tratadas a 10 y 20 min la alcanzaron en 240 min, y la muestra control a los $510 \mathrm{~min}$. De acuerdo a estos resultados el tratamiento de US 30 min presentó una reducción del tiempo de secado del $58.85 \%$, y los de 10 y 20 min del $52.94 \%$. Esto debido a que la aplicación del US genera la creación de microcanales causados por la tensión sistemática, cambia la descompresión del material (llamado "efecto esponja") [2],[33], facilitando la salida de agua de la matriz causando un crecida en las rapidez de secado [2], [50].

La creación de microcanales origina la dispersión y limitación de las células como respuesta a la aplicación de US, sin producir descomposición de las células como lo confirman algunas investigaciones en melón, papaya y piña [34], [35], [36]. De acuerdo a Santacatalina et al. [2], la energía mecánica que el US forma en el transcurso de cavitación, provoca disminución en la resistencia interna y externa a la transmisión de agua en el fruto, con la generación de microcorrientes en las interfases del material sólido facilitando la salida del agua de la matriz vegetal [12].

Otra explicación puede ser que con la aplicación de US se haya incrementado la 
permeabilidad de la cutícula cerosa de la superficie semiesférica de la uchuva, debido a un aumento de los poros por el efecto sónico. De acuerdo a algunos investigadores [37], la cutícula cerosa de algunas frutas presenta permeabilidad baja por los poros pequeños que la conforman, haciendo que el agua circule muy lentamente a través de ellos; es posible que por esta razón la muestra control haya presentado el mayor tiempo de secado.

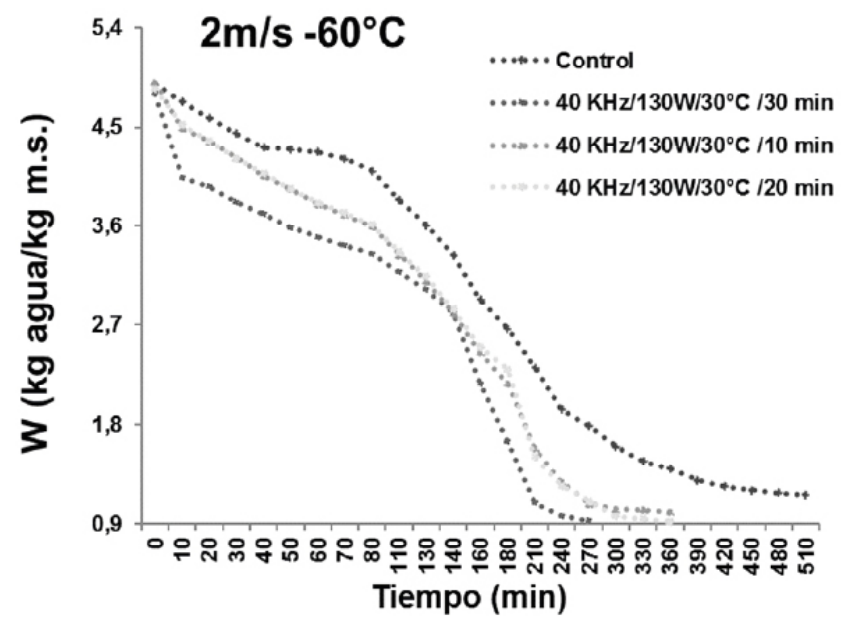

Figura 1. Curvas del contenido de humedad de uchuva pretratada con US $(130 \mathrm{~W} / 40 \mathrm{kHz})$ durante el secado con aire caliente $\left(60^{\circ} \mathrm{C}\right.$ y $\left.2 \mathrm{~m} / \mathrm{s}\right)$. Los valores de la media \pm s.d. $(n=3)$.

Estos resultados presentan comportamiento similar en otros estudios en frutas $y$ vegetales como en fresas que se precisó una consecuencia positiva sobre el tiempo de secado convectivo a $70^{\circ} \mathrm{C}$ y $60 \mathrm{~W}$ de energía, obteniendo disminución del $35 \%$ [38] y del $50 \%$ en el hundimiento de solución de sacarosa [6], en cáscara de limón a $90 \mathrm{~W} / 21.7 \mathrm{kHz}$ disminuyo un $53 \%$ [39]; en papa a $200 \mathrm{~W} / 25$ $\mathrm{kHz}$ la depreciación del lapso de secado fue del $32 \%$ [40]; en manzanas a $50 \mathrm{~W} / 21.9 \mathrm{kHz}$ se alcanzó $55 \%$ para lograr una pérdida de peso del $80 \%$ con relación al peso inicial y de $80,3 \%$ a $-10^{\circ} \mathrm{C}$ y $75 \mathrm{~W}$ [2]; en berenjena a $90 \mathrm{~W}$ una baja del $72 \%$ [32]; en zanahorias fue del $40 \%$ [41]; en hojas de perejil a $21 \mathrm{kHz} / 12 \mathrm{~W} / \mathrm{g}$, fue del 29,8\% [42]; en mora el tiempo de secado redujo en casi cinco veces cuando se trataron con US a 90 micras/20 min [43]. Kowalski et al. [44] manifestaron disminuciones en el tiempo de secado del $79 \%$ para frambuesas.

Wang et al., [51] obtuvieron un incremento en la pérdida de peso del quimbombó o okra exponiéndolo a $25 \mathrm{kHz} / 25{ }^{\circ} \mathrm{C}$ durante 15 minutos; en la granada a $40 \mathrm{kHz} / 100 \mathrm{~W}$ a 30 ${ }^{\circ} \mathrm{C}$ durante 60 y $80 \mathrm{~min}$. Se logró la mayor pérdida de agua [52]. Así mismo, al utilizar como pretratamiento el US en el secado de banano a $80{ }^{\circ} \mathrm{C}$ con velocidad de $3 \mathrm{~m} / \mathrm{s}$, el tiempo de secado se redujo hasta en un $21,6 \%$ y el gasto de energía se redujo en un $22 \%$ [53]; en papa el tiempo de secado se redujo en un $30 \%$ y un $23 \%$ con US + etanol y US + agua respectivamente [54].

Rojas et al. [55] realizaron inmersión de rodajas de manzana en etanol durante $30 \mathrm{~min}$ y US $(21.77 \mathrm{kHz} / 20.5 \mathrm{~kW} / \mathrm{m} 3)$ secadas por convección $\left(50^{\circ} \mathrm{C}, 1 \mathrm{~m} \square \mathrm{s}-1\right)$, se observando una reducción del tiempo de secado convencional del $70 \pm 2 \%$. En calabaza con secado convectivo de $50^{\circ} \mathrm{C}$ y $0.8 \pm 0.1 \mathrm{~m} \square \mathrm{s}-$ la combinación de etanol y US presentaron la mayor reducción tanto en el tiempo de secado $(59 \%)$ como en consumo de energía (44\%) [56].

Determinación del coeficiente de difusividad. Los valores del coeficiente de difusividad efectiva del agua con y sin la aplicación de US se muestran en la tabla 2.

Tabla 2. Valores del coeficiente de difusión efectiva en el secado de uchuva con y sin aplicación de ultrasonido

\begin{tabular}{|c|c|c|c|c|}
\hline \multirow[t]{2}{*}{$\begin{array}{l}\text { Parámetros } \\
\text { estimados }\end{array}$} & \multirow[t]{2}{*}{ Control } & \multicolumn{3}{|c|}{$\begin{array}{c}\text { TRATAMIENTOS } \\
\left(40 \mathrm{KHz} / 130 \mathrm{~W} / 30^{\circ} \mathrm{C}\right)\end{array}$} \\
\hline & & $10 \mathrm{~min}$ & $20 \mathrm{~min}$ & $30 \mathrm{~min}$ \\
\hline $\begin{array}{l}\text { Deff } \quad\left(10^{-10}\right. \\
\left.\mathrm{m}^{2} / \mathrm{s}\right)\end{array}$ & $4.88 \pm 0.43^{a}$ & $6.63 \pm 1.12^{b}$ & $7.13 \pm 1.1^{b}$ & $8.33 \pm 0.87^{c}$ \\
\hline$\%$ VAR & 97.3 & 99.5 & 99.2 & 98.7 \\
\hline
\end{tabular}

Diferentes letras indican que los valores son significativamente diferentes $(p>0,05)$. 
El ajuste del prototipo a los valores experimentales fue mejor en las muestras tratadas con US, con valores del porcentaje de varianza desde 98.7 a $99.5 \%$, mientras que el control mostró $97.3 \%$.

Se observa que el valor Deff se incrementa significativamente $(P<0,05)$ con el aumento del tiempo de exposición del US, desde 4.88 a $8.33 \times 10-10 \mathrm{~m}^{2} / \mathrm{s}$; esto puede deberse al efecto que causa el US al ampliar la energía de calentamiento que aumenta la difusividad del agua [2]. Estos valores de Difusividad están dentro del rango común para la mayoría de los alimentos entre $10-11$ y $10-9 \mathrm{~m}^{2} / \mathrm{s}$ [45]. Puede notarse que los tratamientos con US presentaron mayor valor Deff respecto al tratamiento control, lo que demuestra el potencial del US para apresurar la pérdida de agua en circunstancias de secado; esto debido a la formación de microcanales en la matriz de la uchuva facilitando la movilidad de las moléculas de agua hacia el exterior. De acuerdo a algunos investigadores [38, 46], este incremento del coeficiente de difusión con la aplicación del US está asociado a la variación de las expansiones y contracciones de los ciclos producidos por el poder del US en los materiales, provocando una aceleración del movimiento de agua interior.

Resultados similares en valores de Deff fueron reportados en trabajos previos de vegetales secados con pretratamiento con US como: Fresa [47], manzana [2], papa [40], zanahorias [41]; hojas de perejil [42]; mora [43]. Este efecto se debe probablemente a la variación de las expansiones y contracciones de los ciclos producidos por el poder del US en los materiales, fenómeno que causa una aceleración del movimiento de agua interior, siendo esta manifiesta en el aumento de la Deff [38], [48].

Modelamiento de las cinéticas de secado. La tabla 3 presenta los parámetros cinéticos estimados mediante 5 modelos (Newton,
Henderson and Pabis, Page, Page modificada y logarítmico), incluyendo los criterios de calidad (R2, SSE y x2). El prototipo que representó mejor las curvas de secado experimentales para las tres condiciones estudiadas fue el prototipo Logarítmico; $\mathrm{R}^{2}$ más próximos a 1.0 y SSE y $x^{2}$ más cercanos a cero.

El prototipo que muestra el alto ajuste con los datos experimentales es el logarítmico como se observa en la figura 2; en trabajos previos de secado en cebolla [49] y limón [26] ha sido también el que mejor se ajusta. La tabla 3 muestra que la constante $\mathrm{k}$ se incrementa en todos los modelos cinéticos con el tiempo de aplicación del US, lo que significa que es dependiente de este tiempo.

Tabla 3. Valores de los parámetros de ajuste de los modelos de secado seleccionados.

\begin{tabular}{|c|c|c|c|c|c|}
\hline \multirow[b]{2}{*}{ Modelos } & \multirow{2}{*}{$\begin{array}{l}\text { Pruebas } \\
\text { estadisticas }\end{array}$} & \multirow[t]{2}{*}{ Control } & \multicolumn{3}{|c|}{$\begin{array}{c}\text { TRATAMIENTOS } \\
\left(40 \mathrm{KHz} / 130 \mathrm{~W} / 30^{\circ} \mathrm{C}\right)\end{array}$} \\
\hline & & & US/10min & US/20min & US/30min \\
\hline \multirow{4}{*}{ Newton } & $R^{2}$ & 0.924 & 0.954 & 0.965 & 0.976 \\
\hline & SSE & 0.010 & 0.011 & 0.008 & 0.001 \\
\hline & $x^{2}$ & 0.010 & 0.012 & 0.011 & 0.002 \\
\hline & $k\left(x 10^{-3}\right)$ & $3.20 \pm 0.18$ & $6.10 \pm 0.19$ & $8.56 \pm 1.51$ & $12.02 \pm 1.24$ \\
\hline \multirow{5}{*}{$\begin{array}{c}\text { Henderson } \\
\text { and Pabi }\end{array}$} & $\mathrm{R}^{2}$ & 0.965 & 0.972 & 0.982 & 0.972 \\
\hline & SSE & 0.015 & 0.011 & 0.010 & 0.003 \\
\hline & $\mathrm{X} 2$ & 0.015 & 0.012 & 0.011 & 0.002 \\
\hline & $k\left(x 10^{-3}\right)$ & $4.12 \pm 0.28$ & $6.76 \pm 0.35$ & $8.19 \pm 2.05$ & 12.36 \\
\hline & $n$ & $1.28 \pm 0.04$ & $1.30 \pm 0.01$ & $1.46 \pm$ & $1.12 \pm$ \\
\hline \multirow{5}{*}{ Page } & $\mathrm{R}^{2}$ & 0.983 & 0.991 & 0.992 & 0.995 \\
\hline & SSE & 0.001 & 0.001 & $<0.001$ & 0.001 \\
\hline & $\mathrm{X} 2$ & 0.001 & 0.001 & $<0.001$ & 0.001 \\
\hline & $k\left(x 10^{-4}\right)$ & $8.10 \pm 0.19$ & $9.12 \pm 1.55$ & $11.89 \pm 3.76$ & $25.96 \pm 8.54$ \\
\hline & $\mathrm{n}$ & $1.25 \pm 0.01$ & $1.35 \pm 0.04$ & $1.18 \pm 0.058$ & $1.35 \pm 0.054$ \\
\hline \multirow{5}{*}{$\begin{array}{c}\text { Page } \\
\text { Modificada }\end{array}$} & $\mathrm{R}^{2}$ & 0.985 & 0.991 & 0.993 & 0.987 \\
\hline & SSE & 0.001 & $<0.001$ & $<0.001$ & 0.001 \\
\hline & $\mathrm{X} 2$ & 0.001 & $<0.001$ & $<0.001$ & 0.001 \\
\hline & $k\left(\times 10^{-3}\right)$ & $2.91 \pm 0.11$ & $5.21 \pm 0.21$ & $8.23 \pm 0.46$ & $11.54 \pm 0.34$ \\
\hline & $\mathrm{n}$ & $1.25 \pm 0.01$ & $1.30 \pm 0.01$ & $1.45 \pm 0.06$ & $1.32 \pm 0.05$ \\
\hline \multirow{6}{*}{ Logarítmico } & $R^{2}$ & 0.995 & 0.992 & 0.993 & 0.995 \\
\hline & SSE & $<0.001$ & 0.001 & $<0.001$ & 0.001 \\
\hline & $\mathrm{X} 2$ & $<0.001$ & 0.001 & $<0.001$ & 0.001 \\
\hline & $k\left(\times 10^{-2}\right)$ & $0.14 \pm 0.01$ & $0.35 \pm 0.02$ & $0.61 \pm 0.027$ & $1.01 \pm 0.05$ \\
\hline & $\mathrm{n}$ & $1.56 \pm 0.04$ & $1.32 \pm 0.02$ & $1.31 \pm 0.026$ & $1.079 \pm 0.01$ \\
\hline & a & $\begin{array}{c}- \\
0.51 \pm 0.06\end{array}$ & $\begin{array}{c}- \\
0.21 \pm 0.04\end{array}$ & $-0.14 \pm 0.02$ & $\begin{array}{r}-0.03 \\
\pm 0.01 \\
\end{array}$ \\
\hline
\end{tabular}

El valor $k$ es mayor en los tratamientos con US respecto a la muestra control, lo que podría estar asociado indirectamente a la pérdida de agua de la fruta durante el secado. EI ANOVA 
mostro para todos los parámetros de $\mathrm{k}$ de los modelos cinéticos, a un nivel de confianza del 95\%, diferencias significativas $(P<0,05)$ en la mayoría de los casos, evidenciando que el tiempo de exposición al US afecta significativamente el secado.

\section{Conclusiones}

La aplicación de ultrasonido (40 $\mathrm{KHz} / 130 \mathrm{~W} / 30^{\circ} \mathrm{C} / 10,20$ y $30 \mathrm{~min}$ ) como pretratamiento a al secado convectivo de muestras de uchuvas, evidenció una reducción significativa del tiempo de proceso al compararse con la muestra control; alcanzando la mayor disminución del tiempo de $58.85 \%$ para el tratamiento sometido a 30 min de US. El modelo que mejor predice las cinéticas de secado de uchuva con y sin aplicación de US es el Logaritmo. De acuerdo a estos resultados el uso del ultrasonido sería una alternativa potencial para disminuir el tiempo de secado de la uchuva y de vegetales en general con un ahorro energético significativo.

\section{Referencias}

[1] J. Santacatalina, J. Cárcel, J. Fernández, A. Mulet and J. García, "Impact of applied ultrasonic power on the low temperature drying of apple" Ultrasonics Sonochemistry. vol. 28, pp. 100-109, 2016

[2] L. Mayor and A.M. Sereno, "Modelling shrinkage during convective drying of food materials: a review", Journal of Food Engineering, vol. 61, pp. 373-386, 2004

[3] D.I. Onwude, N. Hashim, K. Abdan, R. Janius, and G. Chen, "The effectiveness of combined infrared and hot-air drying strategies for sweet potato", Journal of Food Engineering, pp.241, 75-87, 2019
[4] Y. Ando, et al., "Improvements of drying rate and structural quality of microwavevacuum dried carrot by freeze-thaw pretreatment", $L w t$, vo. 100, 294-299, 2019

[5] L.Z. Deng, et al., "Chemical and physical pretreatments of fruits and vegetables: Effects on drying characteristics and quality attributes - a comprehensive review", Critical reviews in food science and nutrition, vol. 59, 1408-1432, 2019

[6] J. Garcia-Noguera, I.P. Oliveira, M. Gallão, L. Weller S. Rodrigues y A. Fernandes,"Ultrasound-Assisted Osmotic Dehydration of Strawberries: Effect of Pretreatment Time and Ultrasonic Frequency", Drying Technology. vol. 28, pp. 294-303, 2010

[7] X. Zhou et al., "Effects of infrared radiation drying and heat pump drying combined with tempering on the quality of long-grain paddy rice", International Journal of Food Science \& Technology, vol. 53, 2448-2456, 2018

[8] C. Ortuño, J.V. García-Pérez, J.A. Cárcel, A. Femenía and A. Mulet, "Modeling Ultrasonically Assisted Convective Drying of Eggplant”, Drying Technology, vol. 29, no. 13, pp. 1499-1509, 2010

[9] L. Hassini, S. Azzouz, R. Peczalski, and A. Belghith, "Estimation of potato moisture diffusivity from convective drying kinetics with correlation of shrinkage", Journal of Food Engineering, vol. 79, pp. 47-56, 2007

[10] F. Fernandes, S. Rodrigues, "Application of ultrasound and ultrasound-assisted osmotic dehydration in drying of fruits", Drying Technology, vol. 26, pp. 1509-1516, 2008 


\section{0}

[11] D. Huang, K. Men, D. Li, T. Wen, Z. Gong, B. Sunden and $\mathrm{Z}$. Wu, "Application of ultrasound technology in the drying of food products", Ultrasonics Sonochemistry, VOL. 63, 2020

[12] Y. Zhang and N. Abatzoglou, "Review: fundamentals, applications and potentials of ultrasound-assisted drying", Chemical Engineering Research and Design, vo. 164, pp. 21-46, febrero 2020

[13] J.V. García-Pérez, J.A. Cárcel, E. Riera and A. Mulet, "Influence of the applied acoustic energy on the drying of carrots and lemon peel”, Drying Technology, vol. 27, pp. 281-287, 2009

[14] M. Cortés-Rodriguez, E. Herrera-Herrera and J. Gil-González, "Impregnación de uchuva (Physalis peruviana 1.) De forma semiesférica con una emulsión fortificante", Biotecnología en el Sector Agropecuario y Agroindustrial, vol. 14, no. 1, pp. 27-36, 2016

[15] L. Puente, M. Cortés, C. Pinto-Muñoz, and E. Castro, "Physalis peruvianan Linneaus, The Multiple Properties of a Highly functional fruit: A review", Food Research International, vol. 44, no. 7, pp. 1733-1740, 2011

[16] Instituto Colombiano de Normas Técnica y Certificación (ICONTEC). Norma Técnica Colombiana NTC 4580. Frutas frescas. Uchuva. Especificaciones, Bogotá (Colombia), 1999. [En línea]. Disponible en: https://tienda.icontec. org/wp-content/uploads/pdfs/NTC4580.pdf

[17] A. Castro, L. Rodríguez y E. Vargas, "Secado de uchuva (Physalis peruviana 1) por aire caliente con pretratamiento de osmodeshidratación. VITAE", Revista de la Facultad de Química Farmacéutica, vol. 15, no. 2, pp. 226-231, 2008

[18] S.J. Babalis and V.G. Belessiotis, "Influence of the drying conditions on the drying constants and moisture diffusivity during the thin-layer drying of figs", J. Food Eng, vol. 65, no. 3, pp. 449-458, 2004

[19] J. Crank, The Mathematics of Diffusion, 2nd Ed., Oxford University Press: London, U.K., 1975

[20] A. Vega-Gálvez, L. Puente-Díaz, R. LemusMondaca, and M. Miranda, "Mathematical modeling of thinglayer drying kinetics of cape gooseberry (Physalis peruviana L.)", Journal of Food Processing and Preservation, vol. 38, no. 2, pp. 728-736, 2012

[21] A. Vega-Gálvez, R. Lemus-Mondaca, C. TelloIreland, M. Miranda and F. Yagnam, "Kinetic study of convective drying of blueberry variety O'Neil (Vaccinium corymbosum L.)", Chilean J. Agric. Res, vol. 69, pp. 171-178, 2009

[22] I. Doymaz, "Influence of pretreatment solution on the drying of sour cherry", J. Food Eng, vol. 78, pp. 591-596, 2007

[23] W. Senadeera, B.R. Bhandari, G. Young, and Y.B. Wijesinghe, "Influence of shapes of selected vegetable materials on drying kinetics during fluidized bed drying", J. Food Eng., vol. 58, pp. 277-283, 2003

[24] I.T. Tog Rul, and D. Pehlivan, "Modeling of drying kinetics of simple apricot", J. Food Eng., vol. 58, pp. 23-32, 2003

[25] Akpinar, E.K. Determination of suitable thin layer drying curve model for some vegetables and fruits. J. Food Eng. [Online]. 73, 75-84, 2006. Available at: http://www.sciencedirect. com/science/article/pii/S0260877405000415.

[26] L. Puente-Diaz, E. Echegaray-Pacheco, E. Castro-Montero and K. Di-Scala, "Application of mathemathical models to infrared assisted drying process of lemon waste (citrus limon (1.) 
burm. f. cv. genova)", Dyna, vol. 80, no. 181, pp. 91-97, 2013

[27] C. Duque, L. Alba, V. Villamizar, H. Rafael, P. Giraldo y A. German, "Evaluacion de las tecnicas de secado de uchuva (Physalis peruviana 1.) y mora (Rubus glaucus) con aire caliente y aire caliente microondas", Revista Tumbaga, vol. 6, pp. 17-28, 2011

[28] Y. Ozdemir, A. Ozturk and S.Tüfekçi, "Effect of two dipping pretreatment on drying kinetics of golden berry (Physalis peruviana L.)", African Journal of Agricultural Research, vol. 11, no. 1, pp. 40-47, 2016

[29] G. Yıldız, N. İzli, H. Ünal, and V. Uylaşer, "Physical and chemical characteristics of goldenberry fruit (Physalis peruviana L.)", $J$ Food Sci Technol, vol. 52, no. 4, pp. 2320-2327, 2015

[30] J.E. Vásquez-Parra, C.I Ochoa-Martínez and M. Bustos-Parra, "Effect of chemical and physical pretreatments on the convective drying of cape gooseberry fruits (Physalis peruviana)", Journal of Food Engineering, vol. 119, no. 3, pp. 648-654, 2013

[31] A. Ayala-Aponte, L. Serna-Cock, J. LibrerosTriana, C. Prieto and K. Di Scala, "Influence of osmotic pre-treatment on convective drying of yellow pitahaya", Dyna, vol. 81, no. 188, pp. 145-151. December, 2014

[32] J. García-Pérez, C. Ozuna, C. Ortuño, J. Cárcel and A. Mulet, "Modelling ultrasonically assisted convective drying of eggplant", Drying Technology, vol. 29, no. 13, pp. 1499-1509, 2011

[33] A. Wiktor, M. Sledz, M. Nowacka, K. Rybak and D. Witrowa-Rajchert "The influence of immersion and contact ultrasound treatment on selected properties of the apple tissue", Applied Acoustics, vol. 103, pp. 136-142, 2016
[34] F. Fernandes, M. Gallão y S. Rodrigues, "Effect of osmotic dehydration and ultrasound pretreatment on cell structure: Melon dehydration", LWT - Food Science and Technology, vol. 41, no. 4, pp. 604-610, 2008

[35]F. Fernandes, M. Gallão y S. Rodrigues, "Effect of osmosis and ultrasound on pineapple cell tissue structure during dehydration", Journal of Food Engineering, vol. 90, no. 2, pp. 186-190, 2009

[36] S. Rodrigues, F. Oliveira, M. Gallão y F. Fernandes, " Effect of immersion time in osmosis and ultrasound on papaya cell structure during dehydration", Drying Technolog, vol. 27, no. 2, pp. 220-225, 2009

[37] W. Price, H. Sabarez, R. Storey and P. Back, "Role of the waxi skin layer in moisture loss during dehydration of prunes", Journal of Agricultural and Food Chemistry, vol. 48, no. 9, pp. 4193-4198, 2000

[38] J. Gamboa-Santos, A. Montilla, A. Soria, J. Cárcel, J. García-Pérez and M. Villamiel, "Impact of power ultrasound application on the quality of convective dried Strawberries", Food Chemistry, vol. 161, pp. 40-46, 2014

[39] A. Mulet, J. Cárcel, S. Fuente-Blanco, E. RieraFranco, y J. García-Pérez, "Convective drying of lemon peel assisted by power ultrasound: influence of ultrasonic power applied", Proceedings of the International Congress on Ultrasonics, pp. 1-4, 2007

[40] J. Kroehnke, G. Musielak, "Boratynska, A., Convective drying of potato assisted by Ultrasound", PhD Interdisciplinary Journal, pp. 57-65, 2014

[41] A. Wiktor, M. Dadan, M. Nowacka, K. Rybak and D. Witrowa-Rajchert, "The impact 529 of combination of pulsed electric field and 


\section{2}

ultrasound treatment on air drying kinetics 530 and quality of carrot tissue" Lwt, vol. 110, 7179, 2019

[42] M. Sledz, A. Wiktor, K. Rybak, M. Nowacka and D. Witrowa-Rajchert, "The impact of ultrasound and steam blanching pre-treatments on the drying kinetics, energy consumption and selected properties of parsley leaves", Applied Acoustics, vol. 103, pp. 148-156, 2016

[43] J. Romero and B. Yépez, "Ultrasound as pretreatment to convective drying of Andean blackberry (Rubus glaucus Benth)", Ultrasonics Sonochemistry, vol. 22. pp. 205-210, 2015

[44] S.J. Kowalski, A. Pawłowski, J. Szadzińska, J. Łechtańska, and M. Stasiak, "High power airborne ultrasound assist in combined drying of raspberries", Innov. Food Sci. Emerg. Technol., vol. 34, pp. 225-233, 2016

[45] C. Ozuna, T.G. Álvarez-Arenas, E. Riera, J.V. Cárcel and J.A. Garcia-. Pérez, "Ultrasonics Sonochemistry Influence of material structure on air-borne ultrasonic application in drying", Ultrasonics Sonochemistry, vol. 21, pp. 12351243, 2014

[46] P.S. Madamba, R.H. Griscoll and K.A. Buckle, "The thin-layer drying characteristics of garlic slices", Journal of Food Engineering, vol. 9, no. 1, pp. 75-97, 1996

[47] Y. Campo-Vera, D. Villada, y O. Gélvez, "Efecto De La Aplicación De Ultrasonido Sobre Las Cinéticas De Secado Convectivo De Fresa (Fragaria vesca)", Vitae, vol. 23, no. 1, pp.100 - 104,2016

[48] E. Riera, J. García-Pérez, J. Cárcel, V. Acosta, and J. Gallego-Juárez, Computational study of ultrasound-assisted drying of food materials. Innovative Food Processing Technologies: Advances in Multiphysics Simulation, John
Wiley \& Sons Ltd, pp. 265-301, 2011

[49] G. Sharma, R. Verma and P. Pathare, "Mathematical modeling of infrared radiation thin-layer drying of onion slices", Journal of Food Engineering, vol. 71, no. 3, pp. 282-286, 2005

[50] O. Rodríguez,. et al., "Application of power ultrasound on the convective drying of fruitsand vegetables: effects on quality", Journal of the science of food and agriculture, vol. 99, pp. 966-966, 2019

[51] H. Wang, Q.S. Zhao, X.D. Wang, Z.D. Hong, and B. Zhao, "Pretreatment of ultrasound combined vacuum enhances the convective drying efficiency and physicochemical properties of okra (Abelmoschus esculentus)", Lwt, vol. 112, pp. 108-201. 2019

[52] Z. Allahdad, M. Nasiri, M. Varidi and M. J. Varidi, "Effect of sonication on osmotic dehydration and subsequent air-drying of pomegranate arils", Journal of Food Engineering, vol. 244, 202-211, 2019

[53] F. Nadery Dehsheikh and S. Taghian Dinani, "Coating pretreatment of banana slices using carboxymethyl cellulose in an ultrasonic system before convective drying", Ultrasonics sonochemistry, vol. 52, 401-413, 2019.

[54] M.L. Rojas and P.E.D. Augusto, "Ethanol and ultrasound pre-treatments to improve infrared drying of potato slices", Innovative Food Science \& Emerging Technologies, vol. 49, 6575,2018

[55] M.L. Rojas, P.E.D. Augusto, and J.A. Cárcel, "Ethanol pre-treatment to ultrasound-assisted convective drying of apple", Innovative Food Science \& Emerging Technologies, vol. 102328, doi:10.1016/j.ifset.2020.10232 
[56] M.L. Rojas, I. Silveira and P.E.D. Augusto "Ultrasound and etanol pre-treatments to improve convective drying: Drying, rehydration and carotenoid content of pumpkin", Food and Bioproducts Processing, 2019, doi: https://doi. org/10.1016/j.fbp.2019.10.008 